\title{
Analysis of High Impedance Surface Dimensions on Microstrip Patch Antenna
}

\author{
Vikas kaduskar and Shantanu Jagdale
}

\begin{abstract}
This paper presents a novel design for the implementation of a patch antenna over a high impedance surface. Due to the growth of the wireless communication market, many applications and communication systems require integrated antennas of small size, low cost, low profile, and with enhanced performance characteristics. Unlike normal conductors, this new surface does not support propagating surface waves, and its image currents are not phase reversed. The geometry is analogous to a corrugated metal surface in which the corrugations have been folded up into lumpedcircuit elements, and distributed in a two-dimensional lattice.
\end{abstract}

Index Terms-Corrugated surfaces, high impedance surface, microstrip patch antenna, surface waves.

\section{INTRODUCTION}

Recently, a new kind of ground plane has been developed that is capable of suppressing the propagation of radiofrequency surface currents. This new surface is important for an antenna application because it is has the property of partially isolating the radiating elements from the nearby electromagnetic surroundings. The new surface also provides high electromagnetic surface impedance, which allows the antenna to lie directly adjacent to the ground plane without being shorted out. This allows compact antenna designs where the radiating elements are confined to limited spaces. The high-impedance electromagnetic surface is made of continuous metal, and it conducts dc currents, but it does not conduct ac currents within a forbidden frequency band. The physical geometry consists of a metal sheet, textured with a two-dimensional lattice of resonant elements, which act as a two-dimensional filter to prevent the propagation of electric currents. High impedance Surfaces (HIS) have been frequently employed in electromagnetic devices, as for instance in the design of low-profile antennas or improved electromagnetic absorbers. Repeatedly, it has been shown that HIS structures improve antenna performance and reduce the effects of surface waves.

An MSA in its simplest form consists of a radiating patch on one side of a dielectric substrate and a ground plane on the other side. Conventional microstrip antennas in general have a conducting patch printed on a grounded microwave substrate, and have the attractive features of low profile, light weight, easy fabrication, and conformability to mounting hosts. The radiating patch could be of any

Manuscript received June 28, 2012; revised October 20, 2012.

Vikas kaduskar is with the Electronics Engineering, BVDUCOE, Pune, India (e-mail: vikaskaduskar@gmail.com)

Shantanu Jagdale is with the Department of E \& TC VPCOE, Baramati, India (e-mail: shantanujagdale@gmail.com) arbitrary shape, but it is generally taken as a regular shape for the ease of analysis and understanding of the antenna characteristics. The main advantages of MSAs are listed as follows: They are lightweight and have a small volume and a low-profile planar configuration. They can be made conformal to the host surface. Their ease of mass production using printed-circuit technology leads to a low fabrication cost. They are easier to integrate with other MICs on the same substrate. They allow both linear polarization and CP. They can be made compact for use in personal mobile communication. They allow for dual- and triple-frequency operations.

\section{LITERATURE SURVEY}

The effects of a finite basic High-Impedance Surface (HIS) structure on the impedance, the directivity and the radiation pattern of a printed linear dipole antenna [1]. A high-impedance surface (HIS) is an electrically thin inphase reflector, which provides surface-wave suppression. Within a given frequency band the currents from an adjacent antenna and its image are in phase, as opposed to being $180^{\circ}$ out of phase as typical with conductors. Additionally, because surface waves are suppressed, power loss through the dielectric is minimized. These two properties result in a net increase in radiation efficiency [2]. Two analytical models, namely, the ENG and SD models were investigated for the electromagnetic response of highimpedance surfaces formed by a capacitive array over a grounded dielectric slab perforated with metallic pins [3]. The use of High-Impedance Surfaces (HIS), also known as Electromagnetic Band gap Structures (EBG) to suppress surface waves in antenna applications. Recently. their application has been extended to SSN suppression in PCB's [4]. The analysis of resonant-type antennas based on the fundamental infinite wavelength supported by certain periodic structures is presented in [5]. Since the phase shift is zero for a unit-cell that supports an infinite wavelength, the physical size of the antenna can be arbitrary; the antenna's size is independent of the resonance phenomenon. The antenna's operational frequency depends only on its unit-cell and the antenna's physical size depends on the number of unit-cells. In particular, the unit-cell is based on the composite right/left-handed (CRLH) metamaterial transmission line (TL). It is shown that the CRLH TL is a general model for the required unit-cell, which includes a nonessential series capacitance for the generation of an infinite wavelength

A new type of metallic electromagnetic structure has been developed that is characterized by having high surface impedance. Although it is made of continuous metal, and 
conducts dc currents, it does not conduct ac currents within a forbidden frequency band. Unlike normal conductors, this new surface does not support propagating surface waves, and its image currents are not phase reversed. The geometry is analogous to a corrugated metal surface in which the corrugations have been folded up into lumped-circuit elements, and distributed in a two-dimensional lattice. The surface can be described using solid-state band theory concepts, even though the periodicity is much less than the free-space wavelength. This unique material is applicable to a variety of electromagnetic problems, including new kinds of low-profile antennas [6]. The several different design methodologies for multiband artificial magnetic conducting (AMC) surfaces are presented in [7]. The two important technological novelties aiming at designing antennas that can be integrated into complex systems. First it has introduced a novel metamaterial substrate designed to act as a high impedance slab. Then, it has shown the design of a low profile antenna consisting of a printed dipole located on top of this metamaterial substrate. The high impedance of the substrate permits to locate the metallic dipole very close (sub wavelength distance) to the substrate itself without inhibition of the dipole radiation and its efficiency. Furthermore the substrate inhibits radiation to travel across, resulting in a low profile antenna with high efficiency, even though the antenna system is integrated on top of a lossy structure. The behavior of two realistic feeding structures have also been examined [8].

Major operational disadvantages of Microstrip antennas are their low efficiency, low power, high $Q$ (sometimes in excess of 100), poor polarization purity, poor scan performance, spurious feed radiation and very narrow frequency bandwidth, which is typically only a fraction of a percent or at most a few percent. In some applications, such as in government security systems, narrow bandwidths are desirable. However, there are methods, such as increasing the height of the substrate, that can be used to extend the efficiency (to as large as 90 percent if surface waves are not included) and bandwidth (up to about 35 percent). However, as the height increases, surface waves are introduced which usually are not desirable because they extract power from the total available for direct radiation(space waves). The surface waves travel within the substrate and they are scattered at bends and surface discontinuities, such as the truncation of the dielectric and ground plane, and degrade the antenna pattern and polarization characteristics. Surface waves can be eliminated, while maintaining large bandwidths, by using cavities [9]. The advantages of MSAs make them suitable for numerous applications. The telemetry and communications antennas on missiles need to be thin and conformal and are often MSAs. Radar altimeters use small arrays of Microstrip radiators. Other aircraftrelated applications include antennas for telephone and satellite communications. Microstrip arrays have been used for satellite imaging systems. Patch antennas have been used on communication links between ships or buoys and satellites. Smart weapon systems use MSAs because of their thin profile. Pagers, the global system for mobile communication (GSM), and the global positioning system (GPS) are major users of MSAs [10].

\section{High IMPEDANCE SURFACE}

\section{A. Theory}

By applying a special texture to a conducting surface, it is possible to alter its surface properties. Made of solid metal, the structure conducts DC currents, but over a particular frequency range, it does not conduct AC currents. This new surface is characterized by having high electromagnetic surface impedance. It does not reverse the phase of reflected waves, and the image currents appear in-phase, rather than out-of phase as they are on normal conductors. Furthermore, propagating surface waves are not supported. Instead, radiofrequency currents on the surface radiate efficiently into free space. This high-impedance surface provides a useful new ground plane for novel low profile antennas, and other electromagnetic structures.

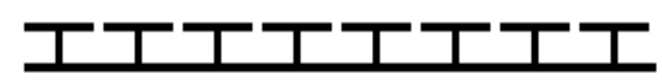

(a)

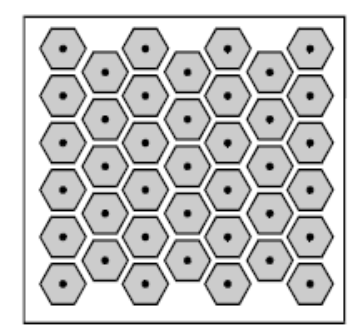

(b)

Fig. 1. (a) Side view of the high-impedance electromagnetic surface. (b) Top view of the high-impedance electromagnetic surface.

The high-impedance properties occur near the resonance frequency $\omega=1 / \sqrt{L C}$ of the surface, where is the effective sheet inductance and is the effective sheet capacitance. The high-impedance electromagnetic surface studied is shown in Fig. 1. It consists of a three-layer printed circuit board, in which the lowest layer is solid metal and the top two layers contain nearly hexagonal metal patches.

\section{B. Lumped Parameter Circuit model}

An equivalent circuit is shown below in Fig. 2 (a). This is the origin of the high electromagnetic surface impedance. Because of its unusual impedance, the surface wave modes on this structure are very different from those on a smooth metal sheet. It can support tightly bound, radio frequency TM modes that propagate much more slowly than the speed of light. It can also support TE modes that are bound to the surface at some frequencies, but radiate readily at other frequencies. In TE surface waves, the electric field is tangential to the surface, and to the propagation direction, while the magnetic field extends out of the sheet in loops. They resemble the TM surface waves described earlier, but with the electric and magnetic fields exchanged, as shown in Fig. 2 (b).

\section{RESEARCH CHALLENGES}

HIS with different dimensions is another area of interest to antenna researchers. To Create A high-impedance surface (HIS) is an electrically thin in-phase reflector, which provides surface-wave suppression. Additionally, because 
surface waves are suppressed, power loss through the dielectric is minimized. These result in a net increase in radiation efficiency. A new type of low profile, lightweight microstrip patch antenna used in combination with a High Impedance surface for improving impedance matching \& bandwidth.

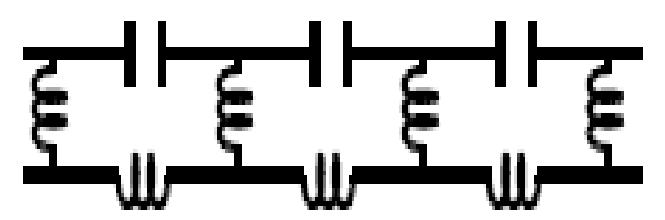

(a)

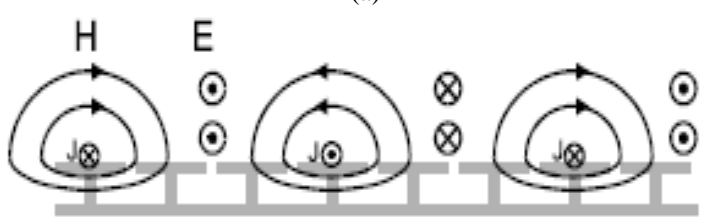

(b)

Fig. 2. (a) An equivalent circuit (b)TE surface wave propagating across HIS

\section{Methodology}

Design a rectangular microstrip patch antenna (RMSA). Radiation pattern plot on Ansoft HFSS.(Check performance of desired patch like beamwidth, gain, return loss etc.)[11]. Design a different dimensions HIS \& implement it with microstrip patch antenna Observe the influence of HIS dimensions on microstrip patch antennas parameters like input impedances, bandwidth etc.

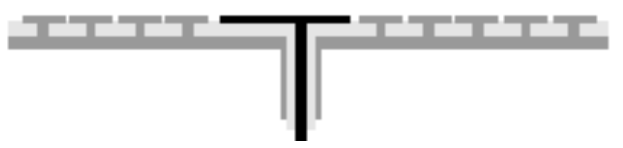

Fig. 3. A patch antenna embedded in a high-impedance ground plane

Surface waves can be suppressed by embedding the patch in a high-impedance ground plane, as shown in Fig. 3. The presence of the nearby metal protrusions tends to raise the resonance frequency of the patch, since the effective cavity volume is reduced. This can be corrected by leaving a small guard ring of bare substrate around the patch, or by increasing the size of the patch. Fig. 4. shows the S11 measurement of two patch antennas - one on an ordinary, metal ground plane, and one on a high-impedance ground plane. This measurement, also called the antenna return loss, is a measure of the amount of power reflected by the antenna toward the generator. A low return loss suggests that the antenna is radiating. In both cases shown, the substrate has a dielectric constant of 10.2, a thickness of $0.625 \mathrm{~mm}$, and a size of $5 \mathrm{~cm}$ square. Circular patches were used, with a diameter of $3.5 \mathrm{~mm}$. A coaxial probe was used to feed the antennas. The patch on the high-impedance ground plane was surrounded by a guard ring consisting of 3 $\mathrm{mm}$ of bare dielectric. The presence of the surrounding metal protrusions tends to confine the electromagnetic fields, reducing the effective size of the cavity, and increasing the resonance frequency slightly.

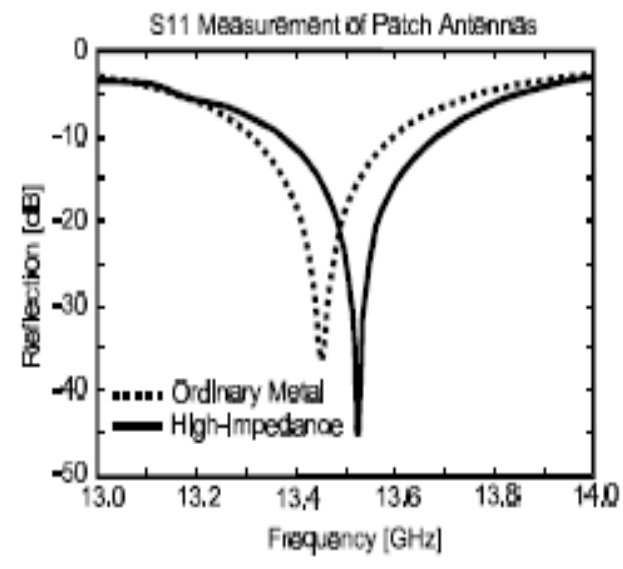

Fig. 4. S11 measurements for patch antennas on two different ground planes

The radiation patterns of the two antennas are shown in Fig. 5. (a) and Fig. 5. (b). The measurements are at a frequency of $13.5 \mathrm{GHz}$, where the two antennas have the same return loss. In both the H-plane and E-plane, the patch on the ordinary, metal ground plane shows significant radiation in the backward direction, and ripples in the forward direction. The pattern is not rotationally symmetric, and is much thinner in the H-plane than in the E-plane. Conversely, the patch on the high-impedance ground plane produces a smooth, symmetric pattern with little backward radiation.

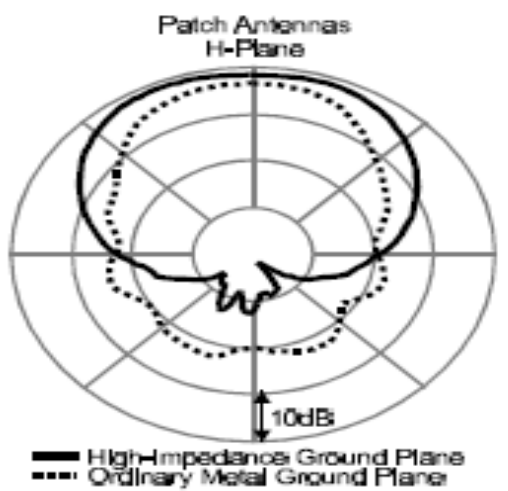

(a)

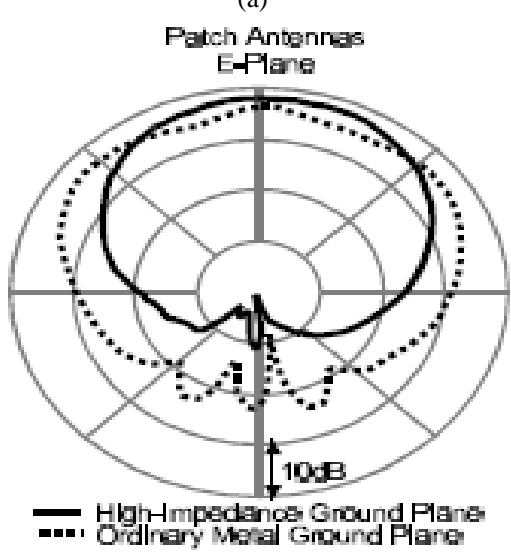

(b)

Fig. 5. (a) H-plane radiation patterns of two patch antennas. (b) E-plane radiation patterns of two patch antennas.

\section{FUTURE WORK}

To observe input impedance and bandwidth for various configurations like hexagonal, square (Fig. 6) and triangular 
of high impedance surfaces over microstrip patch antenna.

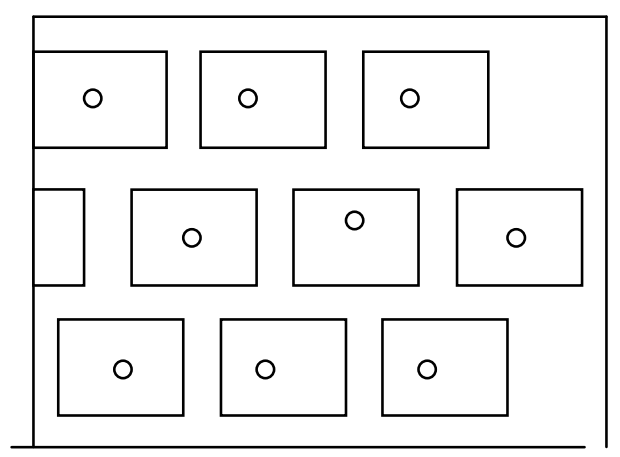

Fig. 6. Square shaped high impedance surface

\section{CONCLUSION}

The radiation pattern of the patch antenna is degraded by surface waves in the same way as the wire monopole. Surface waves radiate from the edges of the ground plane, causing ripples in the antenna pattern, and radiation in the backward direction. If the substrate is thick, or it has a high dielectric constant, the surface wave problem is exacerbated. In future, by employing microstrip patch antenna with different configurations high impedance surfaces, we can achieve better bandwidth, radiation patterns \& input impedance.

\section{REFERENCES}

[1] S. K. Hampel, I. Kiral, O. Schmitz, and I. Rolfes, "Influence of finite high-impedance surface dimensions on the characteristics of a planar printed dipole," European Conference on Antennas and Propagation 2010, Barcelona, pp. 12-16, April 2010.
[2] M. A. Forman, "Reduced-Volume horn antennas with integrated high-impedance electromagnetic surfaces," IEEE Transactions on Antennas and Propagation.

[3] O. Luukkonen, M. G. Silveirinha, A. B. Yakovlev, C. R. Simovski, I. S. Nefedov, and S. A. Tretyakov, "Effects of spatial dispersion on reflection from mushroom-type artificial impedance surfaces," IEEE Trans. Microw. Theory Tech., vol. 57, no. 11, pp. 2692-2699, 2009.

[4] Shahroo: Shahpornia ond Omar M. Romohi, "High-impedance surfaces embedded in printed circuit boards: Design considerations and novel applications," IEEE Transactions on Antennas and Propagation. vol. 13, no. 1, pp. 35691-3372, Apr. 2004.

[5] A. Lai, K. M. K. H. Leong, and T. Itoh, "Infinite wavelength resonant antennas with monopolar radiation pattern based on periodic structures,” IEEE Trans. Antennas Propag., vol. 55, no. 3, pp. 868876, Mar. 2007.

[6] D. Sievenpiper et al., "High-impedance electromagnetic surfaces with a forbidden frequency band," IEEE Transactions on Microwave Theory and Techniques, vol. 47, pp. 2059-2074, Nov. 1999.

[7] J. D. Kern et al., "The design synthesis of multiband artificial magnetic conductors using high impedance frequency selective surfaces,” IEEE Transactions on Antennas and Propagation, vol. 53, pp. 8-17, Jan. 2005.

[8] A. Vallecchi and F. Capolino, "Thin high-impedance metamaterial substrate and its use in low profile antennas suitable for system integration,” Electronic Components and Technology Conference Feb. 2009

[9] C. A. Balanis, Antenna Theory: Analysis and Design, 3rd ed. Wiley Interscience, 2005.

[10] K. P. Ray, Broadband Microstrip Antenna, Artech House,Boston, 2003

[11] K. L. wang, Compact and Broadband Microstrip antenna, John Wiley and son, Inc, 2002.

Vikas Kaduskar was borned in 1986 at pune, completed engineering degree in Electronics \& telecommunication also pursuing Master degree in Digital Systems from Pune University. Currently he is with Bharati Vidyapeeth university college of Engineering as Assistant Professor.

Shantanu Jagdale was borned in 1985. He received Master of Technology degree in 2009. He is currently with Vidyapratishthan college of Engineering, Baramati, India, as Assistant Professor. 